\title{
REVIEW ARTICLE OPEN Molecular mechanisms of inhibiting glucosyltransferases for biofilm formation in Streptococcus mutans
}

\author{
Qiong Zhang $\mathbb{1}^{1}$, Qizhao $\mathrm{Ma}^{1}$, Yan Wang ${ }^{1}$, Hui $\mathrm{Wu}^{2 凶}$ and Jing Zou ${ }^{1 凶}$
}

Glucosyltransferases (Gtfs) play critical roles in the etiology and pathogenesis of Streptococcus mutans (S. mutans)- mediated dental caries including early childhood caries. Gtfs enhance the biofilm formation and promotes colonization of cariogenic bacteria by generating biofilm extracellular polysaccharides (EPSs), the key virulence property in the cariogenic process. Therefore, Gtfs have become an appealing target for effective therapeutic interventions that inhibit cariogenic biofilms. Importantly, targeting Gtfs selectively impairs the $S$. mutans virulence without affecting $S$. mutans existence or the existence of other species in the oral cavity. Over the past decade, numerous Gtfs inhibitory molecules have been identified, mainly including natural and synthetic compounds and their derivatives, antibodies, and metal ions. These therapeutic agents exert their inhibitory role in inhibiting the expression gtf genes and the activities and secretion of Gtfs enzymes with a wide range of sensitivity and effectiveness. Understanding molecular mechanisms of inhibiting Gtfs will contribute to instructing drug combination strategies, which is more effective for inhibiting Gtfs than one drug or class of drugs. This review highlights our current understanding of Gtfs activities and their potential utility, and discusses challenges and opportunities for future exploration of Gtfs as a therapeutic target.

International Journal of Oral Science (2021)13:30

; https://doi.org/10.1038/s41368-021-00137-1

\section{INTRODUCTION}

Dental caries is a multifactorial, sugar-dependent disease driven by the dynamic interactions of cariogenic and commensal microbes within dental plaque (biofilms) formed on tooth surfaces. ${ }^{1,2}$ Although dental biofilms are complex consisting of diverse microbial communities, Streptococcus mutans (S. mutans) has been considered to be the major pathogen in the initiation and development of dental caries, especially in the early childhood caries (ECC). ${ }^{3}$ S. mutans possesses several virulence factors, one of which is the sucrose-dependent adhesion mechanism responsible for dental surface colonization. This adhesion mode contributes significantly to the formation of cariogenic biofilms and cariogenicity. ${ }^{4}$ Sucrose-dependent adhesion is mediated by the activities of glucosyltransferases (Gtfs), a family of enzymes that can split sucrose, the only substrate for the Gtfs, into glucose and fructose, and links the glucose moiety together via glycosidic bonds to form a growing polymer of glucan, termed extracellular polysaccharides (EPSs). The EPS in biofilms provide the microorganisms with a unique microenvironment for their growth, metabolism, and survival, and enables microorganisms to become more resistant to harsh and challenging environmental conditions, host immunity, and traditional antimicrobial therapies, as well as prevents dissociation and enhances its mechanical stability. ${ }^{5}$ More importantly, the interactions between $S$. mutans and certain members of the dental plaque community through Gtfs have recently been shown to exert a major influence on the development and pathogenicity of dental plaque. For example, Veillonella parvula, an oral bacterium that prefers to consume lactic acid as a carbon source, alters
S. mutans virulence factors increasing the expressions of Gtfs in a dual-species biofilm model, suggesting it is engaged in the development of $S$. mutans-mediated dental caries. ${ }^{6}$ In addition to bacteria, fungi can enhance the $S$. mutans pathogenicity via their impact on the expressions of $S$. mutans Gtfs. It has been demonstrated that the cross-kingdom interactions between Candida albicans and S. mutans depend on Gtfs activity, by which increasing the accumulation of $S$. mutans is associated with the development of ECC. ${ }^{7-10}$

S. mutans produces three Gtfs enzymes (GtfB, GtfC, and GtfD), encoded by the $g t f B, g t f C$, and $g t f D$ genes, respectively, of which the expression is distinct but related. $g t f B(4.4 \mathrm{~kb})$ and $g t f C(4.3 \mathrm{~kb})$ are in an operon arrangement separated by $198 \mathrm{bp}$, whose promoters appear to be coordinately regulated, suggesting they can be co-transcribed and are subjected to the similar regulatory mechanism, whereas $g t f D(5.3 \mathrm{~kb})$ is located upstream of the gtfB/ $C$ locus, which has an independent promoter and is not linked to the $g t f B / C$ locus. ${ }^{11,12}$ A similar structure is found in all Gtfs, in which GtfB and GtfC are highly homologous sharing $~ 75 \%$ of amino acid sequences, and GtfD possesses $50 \%$ sequence identity to GtfB and GtfC. All Gtfs have three distinct functional domains: the $\mathrm{N}$-terminal variable junction domain, the highly conserved catalytic domain, and the C-terminal glucan-binding (GB) domain. ${ }^{13,14}$ The activities of Gtfs are mediated through both catalytic and GB functions. ${ }^{15,16}$ Further, Gtfs have a signal peptide comprising about 38 amino acids at the $\mathrm{N}$ terminus, adjacent to which there are about 200 amino acids, a variable domain, which may elucidate the different catalytic functions and binding capacities to different substrates. ${ }^{17}$ Each Gtf plays

\footnotetext{
${ }^{1}$ State Key Laboratory of Oral Diseases, National Clinical Research Center for Oral Diseases and Department of Pediatric Dentistry, West China Hospital of Stomatology, Sichuan University, Chengdu, China and ${ }^{2}$ Department of Integrative Biomedical and Diagnostic Sciences, Oregon Health and Science University School of Dentistry, Portland, OR, USA Correspondence: Hui Wu (wuhu@ohsu.edu) or Jing Zou (zoujing@scu.edu.cn)

These authors contributed equally: Qiong Zhang, Qizhao Ma
}

Received: 18 March 2021 Revised: 29 June 2021 Accepted: 2 September 2021

Published online: 30 September 2021 


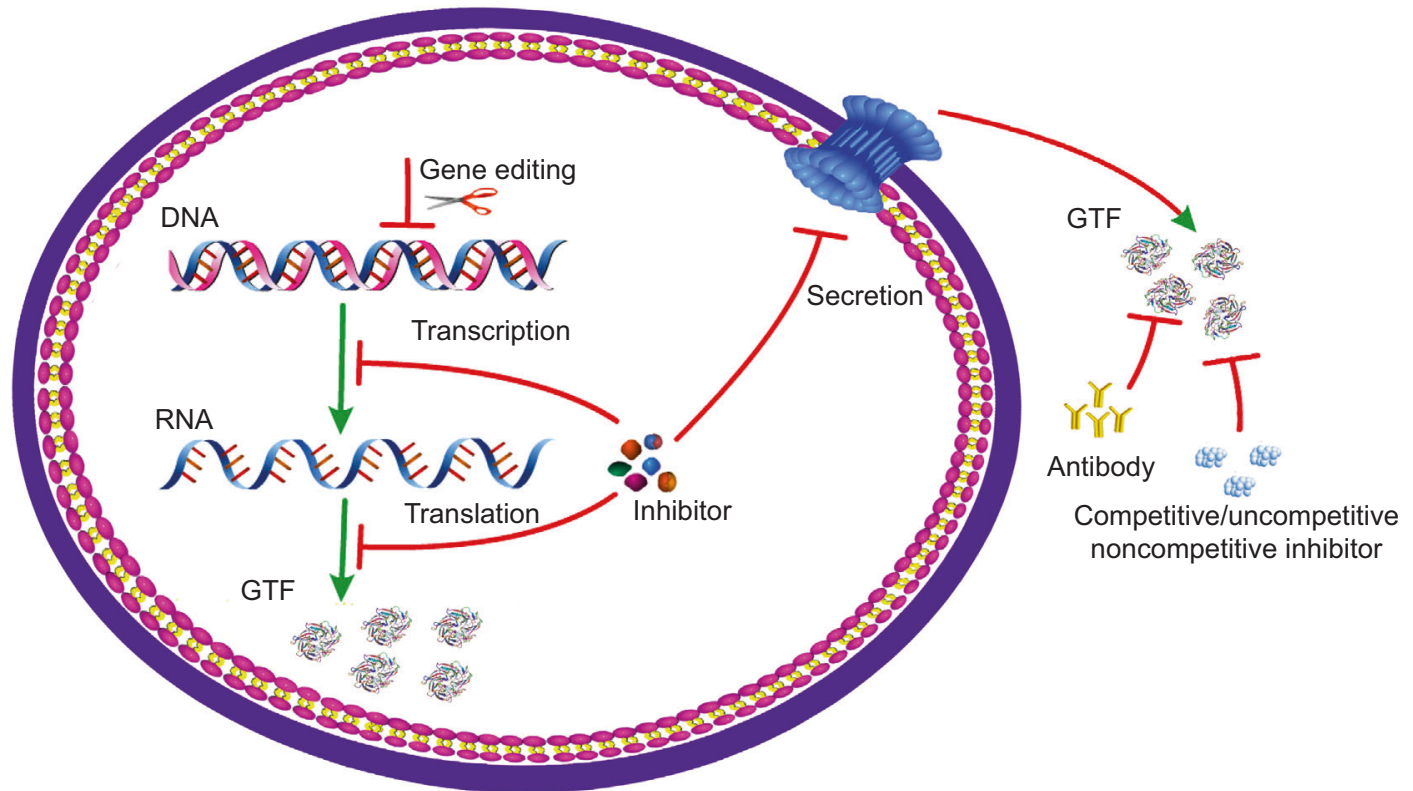

Fig. 1 A diagram of the mechanisms of inhibiting glucosyltransferases for biofilm formation. After Gtfs are synthesized inside the bacteria, they are secreted to the surrounding environment where they are functional. Based on research conducted thus far, the molecular mechanism of current inhibitors against Gtfs could be classified into three categories as follows: 1) modulating the expressions of gtfs genes at the transcriptional level; 2 ) reducing the Gtfs enzymatic activities; 3 ) regulating the secretion of Gtfs outside the cells through dissipating proton motive force across the cell membrane.

different and overlapping roles in the formation of dental biofilms. GtfB (formerly known as Gtfl) synthesizes mostly insoluble glucans containing a-1,3-linked glucose, GtfC (GtfSI) produces a mixture of insoluble and soluble glucans (a-1,6-linked glucose), and GtfD (GtfS) forms predominantly soluble glucans. ${ }^{18}$ Insoluble glucans facilitate bacterial adherence and accumulation on tooth surfaces, and cause biochemical and structural changes in the matrix of the biofilms, which gives rise to the organism's resistance to normal mechanical forces of clearance and affords protection from host immune and non-immune defenses; and soluble glucans may be digested and used as a reserve source of energy when exogenous fermentable carbohydrates are exhausted in the oral cavity, which contributes in part to the low $\mathrm{pH}$ values observed in cariogenic plaques. ${ }^{18,19}$ Furthermore, binding GtfB to bacteria promotes cell clustering and microbial cohesion within plaque biofilms. GtfC exhibits the greatest affinity for saliva-coated hydroxyapatite (sHA) and displays more binding sites than GtfB and GtfD, whereas GtfD displays relatively fewer binding sites and forms soluble glucans, acting as a primer of $\mathrm{GtfB}^{20}$ Therefore, targeting Gtfs through inhibiting their activity and consequently preventing the synthesis of EPS would impair the $S$. mutans virulence without threatening $S$. mutans existence, or the existence of other species in the oral cavity, which is an appealing strategy to treat dental caries when compared to traditional bactericidal treatments. This precision targeting is advantageous in the inhibition of the formation of cariogenic biofilms and bacterial pathogenesis without promoting bacterial antibiotic resistance, while preserving natural bacterial flora of the mouth. ${ }^{21,22}$ Therapeutic agents against Gtfs exert their inhibitory activities at gene and protein levels through distinct molecular mechanisms. Knowledge of the molecular events underlying the inhibition of Gtfs enzymes facilitates the application of inhibitors alone or in combination with agents against various virulence factors, thus developing more potent and selective therapeutics.

Based on research conducted thus far, the molecular mechanism of current inhibitors against the Gtfs activities could be divided into three categories as follows: (1) by modulating the expressions of gtfs genes at the transcriptional level; (2) by reducing the Gtfs enzymatic activities; and (3) by inhibiting the secretion of Gtfs outside the cells through dissipating proton motive force across the cell membrane, which is not unique to the secretion of Gtfs, also to other proteins (Fig. 1 and Table 1). This review provides an overview of diverse active mechanisms, by which promising therapeutic agents disrupt pertinent Gtfs activities in $S$. mutans and concurrently negate $S$. mutansmediated formation of cariogenic biofilms.

\section{MODULATING THE EXPRESSIONS OF GTF GENES}

Targeting upstream signaling pathways

To survive in the continuously changing oral microenvironments, microorganisms use regulatory networks to respond to the stimuli and elicit adaptive responses, which triggers the differential expressions of various regulatory genes. Two-component signal transduction systems (TCSs) are one of the regulatory networks that function as "molecule switches" to modulate the expression of corresponding genes and consequently controlling diverse signaling and metabolic processes including quorum sensing (QS), sporulation, antibiotic/bacteriocin production, and chemotaxis in response to different internal and external stimuli. ${ }^{23-25}$ Typically, TCS works via two regulatory elements consisting of a membraneassociated histidine kinase and a cytoplasmic response regulator. VicRK signaling transduction system, 1 of 13 TCS found in $S$. mutans, consists of VicR (a cytoplasmic response regulator) and VicK (a membrane-associated histidine kinase). It was previously demonstrated that VicK and VicR both positively regulated the expression of $g t f B / C$ by binding their promoter regions. In addition, mutagenesis of the vicK and vicR coding regions affected sucrose-dependent adhesion and biofilm formation. ${ }^{26,27}$ As a gene in the upstream of vicRKX locus, rnc can regulate the expression of vicRKX. Deletion of $r n c$ represses expression of its downstream vicRKX gene through mircroRNA-size small RNAs at the posttranscriptional level and subsequently reduces the expression of $g t f B /$ $C / D{ }^{28,29}$ Cinnamaldehyde, an $a, \beta$-unsaturated aromatic aldehyde, had an inhibitory effect on the expression of vicR, a part of vicRKX TCS in S. mutans. As a result, it reduced the expression of $g t f B / C$ / 
Table 1. Molecules that inhibit gtfs expressions or Gtfs activities

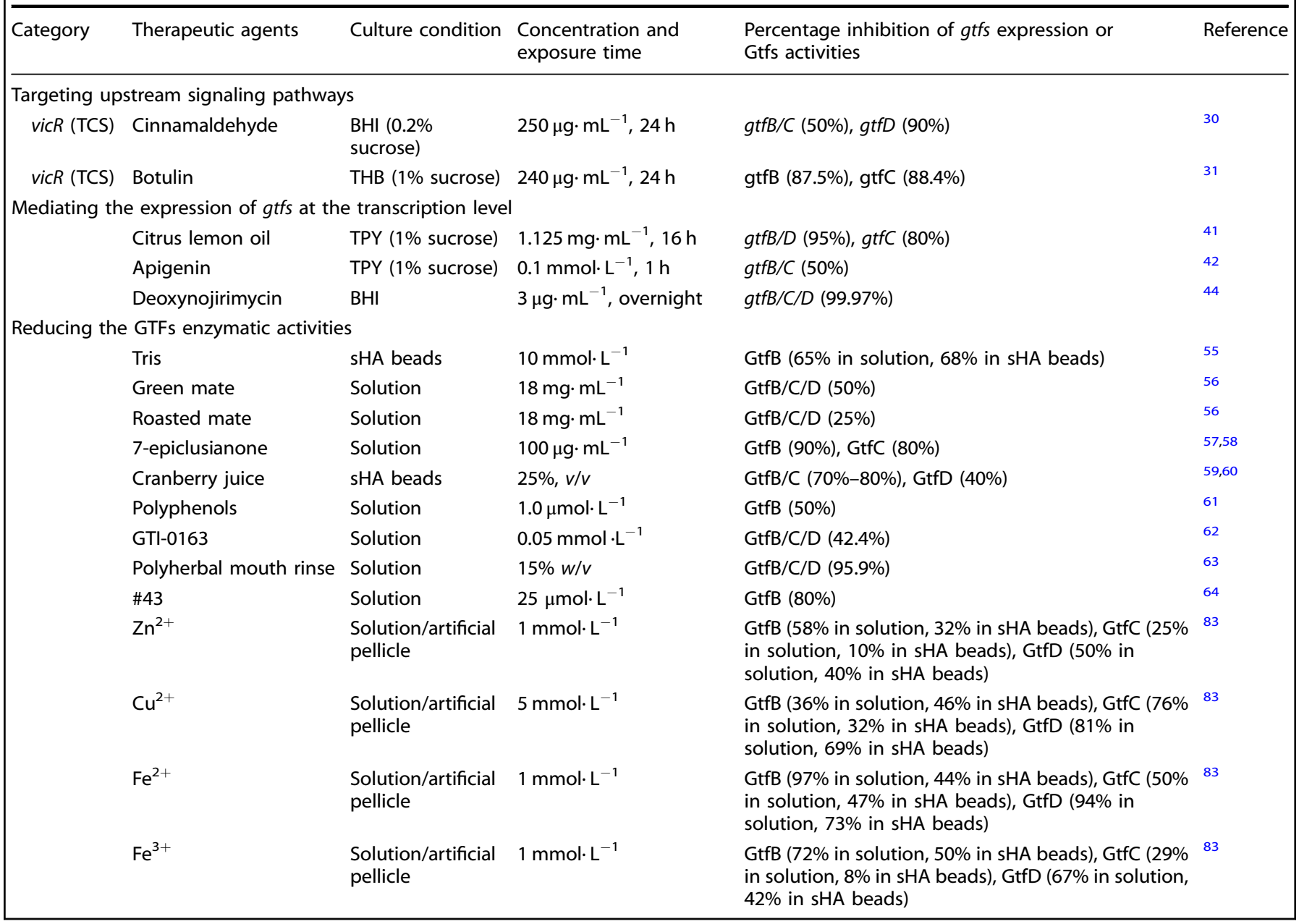

$D .^{30}$ The inhibitory effect on the vicR gene is consistent with those observed in botulin (lup-20(29)-ene-3 $\beta, 28$-diol), a naturally occurring triterpenoid, and botulin $(240 \mu \mathrm{g} / \mathrm{ml})$ reduced the expression of vicR, gtfB, gtfC, and gtfD by 2.4-, 8.4-, 8.6-, and 0.2fold, respectively, compared to the untreated control. ${ }^{31}$ However, the selectivity of these compounds and their mode of action are unknown. RegM, a two-component sensor histidine kinase protein, exhibits high degrees of sequence identity to the catabolite control protein A, a global regulator of sugar metabolism and a primary effector of carbon catabolite repression. RegM could exert its influence on $g t f B / C$ expression through direct interaction with promoter sequences (51 bp upstream of the start codon). ${ }^{32}$

The sugar phosphotransferase system (PTS), the major sugar uptake system in oral streptococci, especially under carbohydratelimiting conditions, is involved in the regulation of gtfs. The PTS consists of two proteins that are common to all of its substrates, enzyme I (EI) and the heat-stable phosphocarrier protein, as well as sugar-specific permease (Ell complexes, consisting of three domains, A, B, C, and sometimes a fourth domain D), which catalyze the transport and concomitant phosphorylation of the substrates. $^{33,34}$ EllAB $^{\text {Man }}$ (encoded by manL), as a part of PTS, modulated the expression of $g t f B / C$ by affecting its promoter. ${ }^{35}$

QS is an intercellular signaling communication network by which bacteria regulate their gene expression in response to environmental cues, such as population density and nutrient availability, by sensing local concentrations of chemical molecules, called autoinducers (Als). ${ }^{36}$ The most common and widespread QS signal among bacteria is Al-2, which is formed by the enzyme S-ribosylhomocysteine lyase (LuxS) that regulates the expression of a large number of genes. ${ }^{37,38}$ As a result, LuxSbased signaling affects $S$. mutans biofilm formation. This is likely due to the altered expression of the $g t f B / C$ genes in the luxS mutant at the mid-log phase. ${ }^{39}$ Recently, it was demonstrated that deletion of smu_833, a putative glycosyltransferase, resulted in a decrease in the transcript level of $g t f B / C$, but not $g t f D$; furthermore, a point mutation within the catalytic DXD motif of smu_833 gave rise to a similar inhibitory effect on the expression of gtfs, suggesting the importance of the glycosyltransferase enzymatic activity of smu_833. However, the exact mechanism by which smu_833 reduced the expression of gtfs is not defined and requires additional research. ${ }^{40}$

Although the expression of gtfs is well regulated by its upstream genes, their specific regulation mechanisms are still unknown. Furthermore, the upstream genes may influence the expression of additional genes. Further research is needed before we have the answer. However, targeting upstream signaling pathways indeed reduces biofilm formation of $S$. mutans by affecting the promoter of $g t f s$ and subsequent expression of $g t f s$, and it does not change cell survival or the homeostasis of oral flora. Therefore, these upstream signaling genes or molecules may be potential targets, which can be used to develop therapeutic agents that prevent and treat dental caries. 
Mediating the expression of $g t f s$ at the transcription level

In addition to targeting upstream signaling pathways or genes, therapeutic agents can directly modulate the expression of gtfs at the transcription level, which has been documented widely in $S$. mutans. These therapeutic agents have been mainly found in natural products or their derivatives. For example, citrus lemon oil (CLO), a natural product, has inhibitory effects on $S$. mutans adherence to glass and saliva-coated enamel surfaces through the inhibition of the transcription of $\mathrm{gtfs}^{41}$ Apigenin, a $4^{\prime}, 5,7-$ trihydroxylflavone commonly identified in plant-derived foods and propolis, decreases the expression of gtfs, especially gtfB and gtfC by $50 \%$ at a concentration of $0.1 \mathrm{mmol} \cdot \mathrm{L}^{-1}$ compared with control, either in solution or absorbed onto sHA beads. ${ }^{42}$ Plant extracts from Symplocarpus renifolius significantly downregulate the expression $g t f B / C / D(2.0-, 1.5-$, and 2.5 -fold change, respectively) at $900 \mu \mathrm{g} \cdot \mathrm{mL}^{-1}$, and plant extracts from Lamium amplexicaule reduce the expression of gtfD (2.0-fold change) at $750 \mu \mathrm{g}$. $\mathrm{mL}^{-1}$, compared with control. ${ }^{43}$ Deoxynojirimycin (DNJ) from traditional fermented foods significantly reduces the expression of gtfs and concurrently decreases the $S$. mutans adherence by $99.97 \%$ under the presence of sugar (1\% glucose), compared with control. ${ }^{44} \mathrm{~A}$ similar inhibitory effect of DNJ on the expression of gtfs was reported by Hasan et al. ${ }^{45}$ In addition, Hasan et al. ${ }^{45}$ investigated the effect of quercitrin, a flavonoid glycoside, and found that quercitrin suppressed the expression of gtfs. More importantly, there was a synergistic effect of anti-cariogenic activity when purified plant-based compounds quercitrin and DNJ are combined, as demonstrated by the significant reduction in the synthesis of both soluble and insoluble polysaccharide. ${ }^{45}$

A great deal of studies has indicated that environmental conditions, such as $\mathrm{pH}$, carbohydrate sources and nutrition availability, and bacterial growth phases mediate the expression of $g t f s$. It is shown that the expression of $g t f B / C$ increased 30 -folds from $48 \mathrm{~h}$ growing biofilm growth to 7 days' growing biofilm when $0.025 \mathrm{~mol} \cdot \mathrm{L}^{-1}$ sucrose was added in culture, which shows that the expression of $g t f B / C$ was significantly different among mature biofilm or relatively young biofilm. This is likely due to the difference in mass transport limitations, the $\mathrm{pH}$, and carbohydrate concentrations experienced by the organisms in various growth environments. ${ }^{19}$ This view was further confirmed in subsequent studies. Environmental $\mathrm{pH}$ and carbohydrate source and availability significantly alter the expression of $g t f B / C .{ }^{46}$ In addition, the expression of gtfs was differentially regulated under various growth phases, which is related to cell-density-dependent factors. ${ }^{47}$ Therefore, it is critical to consider the $S$. mutans living environments when evaluating the inhibitory effects of therapeutic agents. The antibacterial mechanisms governing the regulation of the expression of gtfs are currently under investigation through different targeted and systems biology approaches, such as transcriptomics analysis. The outcomes from these studies may clarify the underlying inhibitory mechanisms that uncover the molecular links between therapeutic agents and the differential expression of $g t f s$.

\section{REDUCING THE GTFS ENZYMATIC ACTIVITIES}

Gene expression studies implicitly showed that changes in mRNA levels have various biological outcomes, presumably mediated by the corresponding changes in protein levels. However, the correlation between gene expression and protein production is not always highly correlated. A variety of posttranscriptional regulatory processes can take place after mRNA is made in controlling steady-state protein abundances, such as mRNA degradation, translational regulation, and protein degradation. ${ }^{48,49}$ To effectively inhibit Gtfs, directly targeting Gtfs activities is a desirable and optimal strategy. A wide range of potential agents has been identified to inhibit Gtfs activities. These various agents inhibited Gtfs activities by different mechanisms, mainly including competitive/noncompetitive/uncompetitive inhibition, and Gtf-based neutralizing antibody approach.

\section{Competitive/uncompetitive/noncompetitive mechanisms of inhibiting Gtfs}

Competitive, uncompetitive, and noncompetitive inhibition are three types of reversible enzyme-inhibitory mechanisms. Inhibitors can prevent a substrate from binding, decrease the enzyme's catalytic activity, or do both. Competitive inhibitors work by binding at the active site due to their structural similarity to its substrate and prevent the binding of the specific enzyme to substrate. Different from competitive inhibitors, uncompetitive inhibitors have a separate binding site on the enzyme and they do not have an affinity for the free enzyme; instead, they only target the intermediate consisting of the enzyme and substrate complex. Similar to uncompetitive inhibitors, the noncompetitive inhibitor binds to the enzyme and the difference is that they bind to an allosteric site that differs from the active site where the substrate binds. ${ }^{50}$ Classic reversible, noncompetitive inhibitors do not affect substrate binding and vice versa. Thus, noncompetitive inhibitors can equally bind enzyme alone or the enzyme-substrate complex. ${ }^{50,51}$ For better elucidating the inhibitory mechanisms, the inhibitors against Gtfs are broadly grouped into the following categories based on their structures and sources, sucrose and its derivatives, and natural and synthetic products.

The Gtfs have a remarkably high degree of specificity for sucrose and agents that are structurally similar to sucrose are thus becoming a structural inspiration to design and develop Gtfs inhibitors. As structural analogs, sucrose derivatives modified at different positions have been demonstrated to have an inhibitory effect on Gtfs activities by various mechanisms, such as 6deoxysucrose, 6-thiosucrose, and 4, 6-dideoxysucrose being competitive inhibitors, sucralose, 4-deoxysucrose, and 4-chloro-4deoxygalactosucrose being noncompetitive inhibitors, and 6, 6'dithiodisucrose as an uncompetitive inhibitor. ${ }^{52-54}$ Natural products also can inhibit Gtfs activities. For example, Tris, an ethanolamine derivative, was found to act as a competitive inhibitor of GtfB and reduced the activity by $65 \%$ at $10 \mathrm{mmol} \cdot \mathrm{L}^{-1}$ compared with control. ${ }^{55}$ Green mate (GM) and roasted mate (RM) water extracts, drinks rich in polyphenolic compounds, reduce glucan polymer synthesis in a competitive manner by serving as strong acceptor substrates for Gtfs, of which RM is more effective. At a concentration of $18 \mathrm{mg} \cdot \mathrm{mL}^{-1}$, the percent inhibition by the RM extraction was about $25 \%$, whereas by GM it was about $50 \% .^{56}$ It has been reported that 7-epiclusianone, isolated from the fruits of Rheedia gardneriana, shows $90 \%$ inhibition of GtfB and $80 \%$ inhibition of GtfC enzymatic activity at a concentration of $100 \mu \mathrm{g} \cdot \mathrm{mL}^{-1}$, compared with control, in a noncompetitive manner and an uncompetitive manner, respectively. ${ }^{57,58}$ In addition, cranberry juice $(25 \%, v / v)$ inhibited surface adsorbed GtfB, GtfC, and GtfD activities (70\%-80\% inhibition of GtfB and $\mathrm{GtfC}$, and $40 \%$ inhibition of GtfD compared to control, respectively). It appears that the inhibition of GtfB and GtfC is more effective than the inhibition of GtfD, which could be attributed in part to the content of flavonols, especially quercetin and its glycosides, and polyphenols. ${ }^{59,60}$ Furthermore, quercetin is a noncompetitive inhibitor of Gtfs $(40 \%-70 \%$ inhibition at a concentration of $500 \mu \mathrm{mol} \cdot \mathrm{L}^{-1}$ compared with control) and polyphenols, extracted from cacao bean husk or oolong tea leaves, were responsible for inhibiting GtfB (50\% inhibition at a concentration of $1.0 \mu \mathrm{mol} \cdot \mathrm{L}^{-1}$ compared with control). ${ }^{61} \mathrm{GTI}-0163$, a mixture of unsaturated fatty acid with a 2:1 ratio of oleic acid to linoleic acid, purified and designated from Prunus salicina, shows a $42.4 \%$ inhibition rate of Gtfs at $0.05 \mathrm{mmol} \cdot \mathrm{L}^{-1}$ in an uncompetitive manner by binding to the enzyme-substrate complex. ${ }^{62}$

Recently, six natural plant products were tested and exhibited considerable levels in inhibiting Gtfs activities. Among them, Terminalia chebula, Psidium guajava, and Pongamia pinnata 
show uncompetitive inhibition, whereas Azadirachta indica displays uncompetitive inhibition, and clove (Syzygium aromaticum) and peppermint oil (Mentha piperita) have allosteric inhibition (sigmoidal response). Moreover, the polyherbal mouth rinse prepared from all the six plants significantly inhibits Gtfs (95.9\%) compared to the chlorohexidine mouthwash's $54 \%$ inhibitory activity. ${ }^{63}$

Although the application of natural products and their derivatives has been documented as one of the most successful approaches for inhibiting activities of Gtfs, some issues need to be resolved, such as the selectivity of these natural compounds is never clear. In this regard, the synthetic products based on the Gtfs structure were designed and developed. \#G43, a smallmolecule inhibitor, screened in silico against the catalytic domain of Gtfs had a potent affinity for GtfC by the interactions of the ortho primary amide group of the compound with key active site residues of GtfC, and thus inhibiting Gtfs activities and S. mutans cariogenicity. ${ }^{64}$ Computer-aided structure-based drug designs guide the development and optimization of lead compounds to increase their affinity, and pharmacodynamic and pharmacokinetic properties. ${ }^{65}$ Strategies employing the in silico methods can be used to predict drug toxicity early on in the drug discovery process, which should facilitate the development of more effective and selective inhibitors.

\section{Gtf-specific antibodies mediated inhibition}

Recognizing the important role of Gtfs in the development of dental biofilms and the pathogenesis of dental caries, developing a vaccine against caries using Gtfs and/or other antigen epitopes as immunogens had been actively pursued and proved to be effective. The approaches include passive immunization involving the use of previously produced antisera elicited by specific Gtf vaccines and active immunization involving the administration of vaccines designed based on targeting immunogenic epitopes of Gtfs.

In passive immunization, ready-made antibodies specific to Gtfs of $S$. mutans are administrated to the host, which should circulate in the blood and provide specific protection against $S$. mutans infection. Antisera are produced using the purified Gtf enzymes, among which antisera to GtfB and GtfC show significant inhibitory effects on both activities regardless of their distribution in solution or adsorbed to a surface, whereas GtfD antisera display more inhibitory effect on GtfD in solution than bound to sHA. One possible explanation for this GtfD unique phenotype is that GtfD in the solution possesses a conformation different from the surface-bound GtfD. ${ }^{66,67}$ Peptides derived from functional motifs within the full-length Gtfs may possess immunogenicity, which has been explored to generate potential vaccines. A self-derived peptide, identical to amino acid sequence 1176-1194 of GtfB, was generated and inhibited GtfB activity in a noncompetitive mode. ${ }^{68}$ To enhance vaccine efficacy, a conjugated antigen containing the two active motifs from different key virulence factors has been constructed and studied. Antibodies against two fusion proteins, consisting of a piece of cell surface protein and GtfB, named PAgA-GB and PACA-GB, respectively, significantly inhibit the $S$. mutans adhesion to sHA beads in the presence or absence of sucrose. PAgA-GB is composed of the alanine-rich region of the cell surface protein antigen (PAgA) and the GB domain of GtfB, while $P A C A-G B$ is composed of the saliva-binding alanine-rich region of the cell surface protein antigen (PACA) and GB of GtfB, respectively. ${ }^{69,70}$ The anticaries efficacy of the PACA-GB antibody was demonstrated in vivo when concentrated immune milk was fed to rats once a day for 55 days. ${ }^{71}$ The inhibitory effects of recombinant protein mentioned above are different in the solution and absorbed onto sHA. However, the sublingual immunization of a recombinant protein consisting of phosphatebinding protein (PstS) has been demonstrated to present dual ability, which not only reduces oral colonization by S. mutans but also inhibits the adhesion to abiotic surface administration. ${ }^{72,73}$

However, passive immunization requires repeated administration of large quantities of antibodies; active immunization, therefore, becomes the focus of active research. Local administration of Gtfs antigen in humans after thorough oral prophylaxis retarded the reaccumulation of $S$. mutans due to the induction of salivary immunoglobulin (SIgA). ${ }^{74}$ Among caries vaccines, the new trend is the use of multigenic DNA/recombinant vaccines, which are safe and stable with stronger antigenicity. For example, a recombinant vaccine combining the $G B$ domain (GLU) of S. mutans GtfB and thioredoxin of Escherichia coli was generated. The vaccine could induce the production of SIgA and reduce colonization and cariogenicity of $S$. mutans in a mice caries model. ${ }^{75}$ Similarly, a fusion anticaries DNA vaccine, pGLUA$P$, encoding two antigen fragments, a GLU fragment of GtfB and A-P fragment of PAC, reduced the levels of dental caries induced by $S$. mutans in rats. ${ }^{76}$ To generate a more persistent and larger amount of antigen, a dual promoter consisting of CMV and nirB promoter driving expression of the genes encoding two virulence factors of $S$. mutans, the saliva-binding region of PAC and the GB region of GtfB. The dual-promoter (CMV-nirB) vaccine generated a large and persistent amount of antigen protein and was superior to nirB promoter used alone in inducing protective immunity against $S$. mutans colonization in BALB/C mice. $^{77}$

In addition, routes of application of vaccines can influence immune responses. Local application to the lower lip area, intranasal, and tonsillar regions showed some promising results. In one study, the application of Gtf vaccine onto the lower lip reduced the indigenous Streptococcal flora compared with a placebo group in young human adults. ${ }^{74}$ Despite these encouraging results, the application of vaccines often requires coadministration of mucosal adjuvants, such as flagellin and enterotoxin from E. coli, Vibrio cholera, and Salmonella typhimurium, which have been successfully used in S. mutans, because adjuvants can accelerate, prolong, or enhance immune responses. ${ }^{78-82}$ However, the potential adverse side effects from these adjuvants are unknown.

Other inhibitory chemicals

Up to now, the inhibitory mechanisms of some effective agents against Gtfs remain to be elucidated. It was reported that CLO, a natural product mixture, effectively reduced the activity of Gtfs (40\%-69\% inhibition at $18 \mathrm{~h}$, the late-exponential phase) in a dose-dependent manner. Although both limonene and $\beta$-pinene, the main components of CLO, have unsaturated bonds in their molecular structure, which may provide binding sites for the sidechain amino acids of Gtfs to diminish its structure stability, future studies are needed to clarify the precise mechanism. ${ }^{41}$ The metal cations and oxidizing compounds have been tested for their ability to inhibit the activities of Gtfs. The results showed that the ability to inhibit Gtfs depends on the Gtfs status, whether they are in solution or adsorbed to the experimental pellicle. In solution, the metal ions including $\mathrm{Zn}^{2+}, \mathrm{Cu}^{2+}, \mathrm{Fe}^{2+}$, and $\mathrm{Fe}^{3+}$, and the oxidizing compounds such as Rose Bengal and hypochlorite significantly inhibit Gtfs activities. In contrast, Gtfs adsorbed to sHA beads exhibit greater resistance to the above inhibitors. ${ }^{83}$ The discrepancy may be due to the conformational changes in the surface attachment resulting in modification of Gtf active sites. Although the exact mechanism by which metal ions inhibit the Gtfs is currently unknown, it has been proposed that metal ions are capable of inactivating Gtf enzymatic activities via covalent modification of amino-acid sulphydryl groups. Reacting with oxygen, these metal ions may oxidatively and covalently modify the Gtfs structure. ${ }^{83}$ 


\section{INFLUENCES ON SECRETION OF GTFS BY DISSIPATING PROTON MOTIVE FORCE ACROSS THE CELL MEMBRANE}

In $S$. mutans, multiple routes exist for Gtfs secretion, including membrane vesicles, protein secretory (Sec) pathway, and the proton motive force. ${ }^{84-87}$ Among these secretory routes, inhibitors against the proton motive force have been well identified. The proton motive force is required in all bacteria to grow and remain viable under replicating and non-replicating conditions. Metabolic energy is conserved in bacteria by an electrochemical proton gradient across a proton-impermeable membrane. ${ }^{88}$ The electron transport chain components are membrane-bound across the membrane to achieve the import of protons from the cytoplasm and release them to the outside the cell. The proton motive force consists of two gradients: an electrical potential $(\Delta \psi$, positive outside $/$ negative $_{\text {inside }}$ ) and a chemical transmembrane gradient of protons $\left(\Delta \mathrm{pH}\right.$, acidic outside/alkaline $\left._{\text {inside }}\right) .{ }^{89-92}$ Enzyme secretion by bacterial cells, in general, is coupled to the proton motive force. Some drugs can reduce the proton motive force by enhancing proton permeability and discharging $\Delta \mathrm{pH}$ across the cell membrane; ${ }^{93-95}$ thus, the side effects and selectivity are not defined.

Fluoride is a well-studied and widely used agent for the prevention of dental caries by inhibiting demineralization and promote remineralization of the teeth and early caries lesions. ${ }^{96}$ Moreover, it has been shown that fluoride has multiple effects on bacteria metabolism and can inhibit bacterial enzymatic activity, which is related to its interference with the transport either directly by dissipating $\Delta \mathrm{pH}$ or, less directly, by inhibiting the membrane-associated proton-pumping $\mathrm{H}^{+}$-ATPase as the organisms attempt to meet the increased demand for proton excretion. ${ }^{97-99}$ It has been demonstrated that fluoride can partially inhibit the secretion of Gtfs by dissipating $\Delta \mathrm{pH}$, the proton motive force, across the cell membrane at low concentrations (up to 3.8 p.p.m.) and then modulates the formation of EPS in S. mutans. ${ }^{100,101}$

In addition, tt-farnesol, a 3,7,11-trimethyl-2,6,10-dodecatrien-1ol, affects the synthesis of EPSs by diminishing the proton motive force across the cell membrane and reducing the secretion of Gtfs. ${ }^{102}$ These agents exert inhibitory effects through impairing secretion function, not decreasing the expression of Gtfs. Therefore, when combined with additional cariostatic agents inhibiting the expression of Gtfs, it synergistically enhances their effectiveness. The fact is that topical application of apigenin and tt-farnesol $\left(1.33 \mathrm{mmol} \cdot \mathrm{L}^{-1} \mathrm{each}\right)$, both identified in propolis, in combination diminishes the incidence of smooth-surface caries (up to $60 \%$ reduction) and displays lower smooth-surface caries severity than either compound alone. ${ }^{103}$ Similar enhanced effectiveness has been demonstrated in the combination of fluoride and other agents. The combination of apigenin, tt-farnesol, and fluoride was the more effective treatment in reducing the biomass and insoluble glucans of S. mutans biofilms than apigenin and fluoride, and $\mathrm{tt}$-farnesol and fluoride through different mechanisms: apigenin inhibiting the expression of Gtfs, and tt-farnesol and fluoride both affecting the secretion of Gtfs. ${ }^{102}$

\section{CONCLUSIONS AND FUTURE PERSPECTIVES}

Recognizing the crucial role of Gtfs in the development of dental biofilms and the pathogenesis of dental caries, interference with Gtfs sparked remarkable interests among alternatives for drug development. Importantly, this approach is more advantageous in reducing the expression of the virulence factors of $S$. mutans without suppressing the resident oral flora. We have broadly categorized these agents according to their inhibitory mechanisms: modulating the expression of gtf genes, affecting the Gtfs activities, and reducing the secretion of Gtfs.

Among these Gtfs inhibitors, natural products have long been recognized as an important source of therapeutic medicines. Current challenges and difficulties in the application of natural products include lack of standard procedures, isolation of pure chemical products or compounds, and elucidation of modes of action, owing to their complex chemistry and variable composition, as well as unknown interactions between compounds and Gtfs active sites. Apart from natural compounds, synthetic products, antibodies, and metal ions have also been recognized in inhibiting Gtfs activities; however, their inhibitory mechanisms are not known and still under investigation. The convergence of new analytical technologies with advances in synthetic drug-like small-molecule compounds targeting Gtfs using computer-aided designs and innovative isolation and preparation methodologies has opened the door to a new era in the development of novel anticaries therapies and the understanding regulatory mechanisms. ${ }^{104-111}$ The combinational therapeutics of different inhibitors including multigenic DNA/recombinant vaccines have shown significantly improved anti-cariogenic efficacy, a promising strategy to design and develop anticaries agents.

In summary, Gtfs are the crucial virulence factors in the etiology and pathogenesis of dental caries, which provides a key target to the discovery of effective inhibitors that affect gene expression or/ and enzyme activity, or secretion. Inhibiting Gtfs led to the reduction in $S$. mutans adhesion and accumulation on the tooth surfaces, and hence the formation of dental biofilms. However, different therapeutic strategies display diverse selectivity and sensitivity by different modes of action. Therefore, understanding new underlying molecular mechanisms of inhibiting Gtfs is critical for developing anticaries agents that should guide the combination of agents targeting additional virulence factors.

\section{ACKNOWLEDGEMENTS}

This research was supported by the National Natural Science Foundation of China (82170947), the Applied Basic Research Project of Science and Technology Department of Sichuan Province (2020YJ0296), and the Innovation and Collaborative Project of Science and Technology Department of Sichuan Province (2019YFH0025).

\section{AUTHOR CONTRIBUTIONS}

H.W. and J.Z. conceived and designed the structure of this review. Q.Z., Q.M., and Y.W. wrote the manuscript. H.W. and J.Z. revised the paper. Q.Z. and Q.M. are co-first authors and they contributed equally to this work.

\section{ADDITIONAL INFORMATION}

Competing interests: The authors declare no competing interests.

\section{REFERENCES}

1. Fejerskov, O. Changing paradigms in concepts on dental caries: consequences for oral health care. Caries Res. 38, 182-191 (2004).

2. Marsh, P. D. Are dental diseases examples of ecological catastrophes? Microbiology 149, 279-294 (2003).

3. Wang, Y., Lee, S. M. \& Dykes, G. A. Potential mechanisms for the effects of tea extracts on the attachment, biofilm formation and cell size of Streptococcus mutans. Biofouling 29, 307-318 (2013).

4. Banas, J. A. Virulence properties of Streptococcus mutans. Front. Biosci. 9, 1267-1277 (2004).

5. Simón-Soro, A. \& Mira, A. Solving the etiology of dental caries. Trends Microbiol. 23, 76-82 (2015).

6. Liu, S. et al. Effect of Veillonella parvula on the physiological activity of Streptococcus mutans. Arch. Oral. Biol. 109, 104578 (2020).

7. Gregoire, S. et al. Role of glucosyltransferase B in interactions of Candida albicans with Streptococcus mutans and with an experimental pellicle on hydroxyapatite surfaces. Appl. Environ. Microbiol. 77, 6357-6367 (2011).

8. Hwang, G., Marsh, G., Gao, L., Waugh, R. \& Koo, H. Binding force dynamics of Streptococcus mutans-glucosyltransferase B to Candida albicans. J. Dent. Res. 94, 1310-1317 (2015).

9. Kim, D. et al. Candida albicans stimulates Streptococcus mutans microcolony development via cross-kingdom biofilm-derived metabolites. Sci. Rep. 7, 41332 (2017). 
10. Yang, C. et al. Antigen I/II mediates interactions between Streptococcus mutans and Candida albicans. Mol. Oral. Microbiol. 33, 283-291 (2018).

11. Fujiwara, T. et al. Molecular analyses of glucosyltransferase genes among strains of Streptococcus mutans. FEMS Microbiol. Lett. 161, 331-336 (1998)

12. Wexler, D. L., Hudson, M. C. \& Burne, R. A. Streptococcus mutans fructosyltransferase ( $\mathrm{ftf}$ ) and glucosyltransferase ( $\mathrm{gtfBC}$ ) operon fusion strains in continuous culture. Infect. Immun. 61, 1259-1267 (1993).

13. Kralj, S. et al. Glucan synthesis in the genus Lactobacillus: isolation and characterization of glucansucrase genes, enzymes and glucan products from six different strains. Microbiology 150, 3681-3690 (2004).

14. Monchois, V., Willemot, R. M. \& Monsan, P. Glucansucrases: mechanism of action and structure-function relationships. FEMS Microbiol. Rev. 23, 131-151 (1999).

15. Koga, T., Oho, T., Shimazaki, Y. \& Nakano, Y. Immunization against dental caries. Vaccine 20, 2027-2044 (2002).

16. Smith, D. J. Dental caries vaccines: prospects and concerns. Crit. Rev. Oral. Biol. Med. 13, 335-349 (2002).

17. van Hijum, S. A., Kralj, S., Ozimek, L. K., Dijkhuizen, L. \& van Geel-Schutten, I. G. Structure-function relationships of glucansucrase and fructansucrase enzymes from lactic acid bacteria. Microbiol. Mol. Biol. Rev. 70, 157-176 (2006).

18. Paes Leme, A. F., Koo, H., Bellato, C. M., Bedi, G. \& Cury, J. A. The role of sucrose in cariogenic dental biofilm formation-new insight. J. Dent. Res. 85, 878-887 (2006).

19. Burne, R. A., Chen, Y. Y. \& Penders, J. E. Analysis of gene expression in Streptococcus mutans in biofilms in vitro. Adv. Dent. Res. 11, 100-109 (1997).

20. Vacca-Smith, A. M. \& Bowen, W. H. Binding properties of streptococcal glucosyltransferases for hydroxyapatite, saliva-coated hydroxyapatite, and bacterial surfaces. Arch. Oral. Biol. 43, 103-110 (1998).

21. Cegelski, L., Marshall, G. R., Eldridge, G. R. \& Hultgren, S. J. The biology and future prospects of antivirulence therapies. Nat. Rev. Microbiol. 6, 17-27 (2008).

22. Zhu, F., Zhang, H. \& Wu, H. Glycosyltransferase-mediated sweet modification in oral Streptococci. J. Dent. Res. 94, 659-665 (2015).

23. Stein, T. et al. Dual control of subtilin biosynthesis and immunity in Bacillus subtilis. Mol. Microbiol. 44, 403-416 (2002).

24. Stock, A. M., Robinson, V. L. \& Goudreau, P. N. Two-component signal transduction. Annu. Rev. Biochem. 69, 183-215 (2000).

25. Strauch, M. A. \& Hoch, J. A. Signal transduction in Bacillus subtilis sporulation. Curr. Opin. Genet. Dev. 3, 203-212 (1993).

26. Senadheera, M. D. et al. A VicRK signal transduction system in Streptococcus mutans affects gtfBCD, gbpB, and ftf expression, biofilm formation, and genetic competence development. J. Bacteriol. 187, 4064-4076 (2005).

27. Senadheera, M. D. et al. The Streptococcus mutans vicX gene product modulates $\mathrm{gtfB} / \mathrm{C}$ expression, biofilm formation, genetic competence, and oxidative stress tolerance. J. Bacteriol. 189, 1451-1458 (2007).

28. Mao, M.-Y. et al. The regulator gene rnc is closely involved in biofilm formation in Streptococcus mutans. Caries Res. 52, 347-358 (2018).

29. Mao, M. Y. et al. The rnc gene promotes exopolysaccharide synthesis and represses the vicRKX gene expressions via microRNA-size small RNAs in Streptococcus mutans. Front. Microbiol. 7, 687 (2016).

30. He, Z., Huang, Z., Jiang, W. \& Zhou, W. Antimicrobial activity of cinnamaldehyde on Streptococcus mutans biofilms. Front. Microbiol. 10, 2241 (2019).

31. Viszwapriya, D., Subramenium, G. A., Radhika, S. \& Pandian, S. K. Betulin inhibits cariogenic properties of Streptococcus mutans by targeting vicRK and gtf genes. Antonie Van Leeuwenhoek 110, 153-165 (2017).

32. Browngardt, C. M., Wen, Z. T. \& Burne, R. A. RegM is required for optimal fructosyltransferase and glucosyltransferase gene expression in Streptococcus mutans. FEMS Microbiol. Lett. 240, 75-79 (2004).

33. Lengeler, J. W., Jahreis, K. \& Wehmeier, U. F. Enzymes II of the phospho enol pyruvate-dependent phosphotransferase systems: their structure and function in carbohydrate transport. Biochem. Biophys. Acta Bioenergetics 1188, 1-28 (1994).

34. Postma, P. W., Lengeler, J. W. \& Jacobson, G. R. Phosphoenolpyruvate:carbohydrate phosphotransferase systems of bacteria. Microbiol. Rev. 57, 543-594 (1993).

35. Abranches, J., Chen, Y. Y. \& Burne, R. A. Characterization of Streptococcus mutans strains deficient in EIIAB man of the sugar phosphotransferase system. Appl. Environ. Microbiol. 69, 4760-4769 (2003).

36. Shin, N. R., Lee, D. Y. \& Yoo, H. S. Identification of quorum sensing-related regulons in Vibrio vulnificus by two-dimensional gel electrophoresis and differentially displayed reverse transcriptase PCR. FEMS Immunol. Med. Microbiol. 50, 94-103 (2007).

37. Sztajer, $\mathrm{H}$. et al. Autoinducer-2-regulated genes in Streptococcus mutans UA159 and global metabolic effect of the luxS mutation. J. Bacteriol. 190, 401-415 (2008).

38. Wen, Z. T. et al. Transcriptome analysis of LuxS-deficient Streptococcus mutans grown in biofilms. Mol. Oral. Microbiol. 26, 2-18 (2011).
39. Yoshida, A., Ansai, T., Takehara, T. \& Kuramitsu, H. K. LuxS-based signaling affects Streptococcus mutans biofilm formation. Appl. Environ. Microbiol. 71, 2372-2380 (2005).

40. Rainey, K., Michalek, S. M., Wen, Z. T. \& Wu, H. Glycosyltransferase-mediated biofilm matrix dynamics and virulence of Streptococcus mutans. Appl. Environ. Microbiol. 85, e02247-02218 (2019).

41. Liu, Y. et al. Effect of citrus lemon oil on growth and adherence of Streptococcus mutans. World J. Microbiol. Biotechnol. 29, 1161-1167 (2013).

42. Koo, H. et al. Influence of apigenin on gtf gene expression in Streptococcus mutans UA159. Antimicrob. Agents Chemother. 50, 542-546 (2006).

43. Lee, Y. C., Cho, S. G., Kim, S. W. \& Kim, J. N. Anticariogenic potential of Korean native plant extracts against Streptococcus mutans. Planta Med. 85, 1242-1252 (2019).

44. Yoo, Y. et al. Inhibitory effect of Bacillus velezensis on biofilm formation by Streptococcus mutans. J. Biotechnol. 298, 57-63 (2019).

45. Hasan, S., Singh, K., Danisuddin, M., Verma, P. K. \& Khan, A. U. Inhibition of major virulence pathways of Streptococcus mutans by quercitrin and deoxynojirimycin: a synergistic approach of infection control. PLOS ONE 9, e91736 (2014).

46. Li, Y. \& Burne, R. A. Regulation of the gtfBC and $\mathrm{ftf}$ genes of Streptococcus mutans in biofilms in response to $\mathrm{pH}$ and carbohydrate. Microbiology 147 2841-2848 (2001).

47. Goodman, S. D. \& Gao, Q. Characterization of the gtfB and gtfC promoters from Streptococcus mutans GS-5. Plasmid 43, 85-98 (2000).

48. Liu, Y., Beyer, A. \& Aebersold, R. On the dependency of cellular protein levels on mRNA abundance. Cell 165, 535-550 (2016).

49. Vogel, C. \& Marcotte, E. M. Insights into the regulation of protein abundance from proteomic and transcriptomic analyses. Nat. Rev. Genet. 13, 227-232 (2012).

50. Ring, B., Wrighton, S. A. \& Mohutsky, M. Reversible mechanisms of enzyme inhibition and resulting clinical significance. Methods Mol. Biol. 1113, 37-56 (2014).

51. Whiteley, C. G. Mechanistic and kinetic studies of inhibition of enzymes. Cell Biochem. Biophys. 33, 217-225 (2000).

52. Binder, T. P. \& Robyt, J. F. Inhibition of Streptococcus mutans 6715 glucosyltransferases by sucrose analogs modified at positions 6 and 6 '. Carbohydr. Res. 140, 9-20 (1985).

53. Tanriseven, A. \& Robyt, J. F. Synthesis of 4,6-dideoxysucrose, and inhibition studies of Leuconostoc and Streptococcus D-glucansucrases with deoxy and chloro derivatives of sucrose modified at carbon atoms 3, 4, and 6. Carbohydr. Res. 186, 87-94 (1989).

54. Young, D. A. \& Bowen, W. H. The influence of sucralose on bacterial metabolism. J. Dent. Res. 69, 1480-1484 (1990).

55. Wright, W. G., Thelwell, C., Svensson, B. \& Russell, R. R. Inhibition of catalytic and glucan-binding activities of a streptococcal GTF forming insoluble glucans. Caries Res. 36, 353-359 (2002).

56. Battagim, J. et al. Comparative study of the effect of green and roasted water extracts of mate (llex paraguariensis) on glucosyltransferase activity of Streptococcus mutans. J. Enzym. Inhib. Med. Chem. 27, 232-240 (2012).

57. Branco-de-Almeida, L. S. et al. Effects of 7-epiclusianone on Streptococcus mutans and caries development in rats. Planta Med. 77, 40-45 (2011).

58. Murata, R. M. et al. Inhibitory effects of 7-epiclusianone on glucan synthesis, acidogenicity and biofilm formation by Streptococcus mutans. FEMS Microbiol. Lett. 282, 174-181 (2008).

59. Koo, H., Nino de Guzman, P., Schobel, B. D., Vacca Smith, A. V. \& Bowen, W. H. Influence of cranberry juice on glucan-mediated processes involved in Streptococcus mutans biofilm development. Caries Res. 40, 20-27 (2006).

60. Koo, H., Rosalen, P. L., Cury, J. A., Park, Y. K. \& Bowen, W. H. Effects of compounds found in propolis on Streptococcus mutans growth and on glucosyltransferase activity. Antimicrob. Agents Chemother. 46, 1302-1309 (2002).

61. Nakahara, K. et al. Inhibitory effect of oolong tea polyphenols on glycosyltransferases of mutans Streptococci. Appl. Environ. Microbiol. 59, 968-973 (1993).

62. Won, S. R. et al. Oleic acid: an efficient inhibitor of glucosyltransferase. FEBS Lett. 581, 4999-5002 (2007).

63. Mandava, K. et al. Design and study of anticaries effect of different medicinal plants against S.mutans glucosyltransferase. BMC Complement. Alter. Med. 19, 197 (2019).

64. Zhang, Q. et al. Structure-based discovery of small molecule inhibitors of cariogenic virulence. Sci. Rep. 7, 5974 (2017).

65. Hartman, A. M. et al. Potential dental biofilm inhibitors: dynamic combinatorial chemistry affords sugar-based molecules that target bacterial glucosyltransferase. ChemMedChem 16, 113-123 (2021).

66. Kopec, L. K., Vacca-Smith, A. M. \& Bowen, W. H. Structural aspects of glucans formed in solution and on the surface of hydroxyapatite. Glycobiology 7, 929-934 (1997).

67. Wunder, D. \& Bowen, W. H. Effects of antibodies to glucosyltransferase on soluble and insolubilized enzymes. Oral. Dis. 6, 289-296 (2000). 
68. Eto, A. et al. Inhibitory effect of a self-derived peptide on glucosyltransferase of Streptococcus mutans. Possible ovel anticaries measure. J. Biol. Chem. 274, 15797-15802 (1999).

69. Kawato, T., Yamashita, Y., Katono, T., Kimura, A. \& Maeno, M. Effects of antibodies against a fusion protein consisting of parts of cell surface protein antigen and glucosyltransferase of Streptococcus sobrinus on cell adhesion of mutans streptococci. Oral. Microbiol. Immunol. 23, 14-20 (2008).

70. Oho, T. et al. Bovine milk antibodies against cell surface protein antigen PAcglucosyltransferase fusion protein suppress cell adhesion and alter glucan synthesis of Streptococcus mutans. J. Nutr. 129, 1836-1841 (1999).

71. Mitoma, M. et al. Passive immunization with bovine milk containing antibodies to a cell surface protein antigen-glucosyltransferase fusion protein protects rats against dental caries. Infect. Immun. 70, 2721-2724 (2002).

72. Ferreira, E. L. et al. Sublingual immunization with the phosphate-binding-protein (PstS) reduces oral colonization by Streptococcus mutans. Mol. Oral. Microbiol. 31, 410-422 (2016).

73. Luz, D. E., Nepomuceno, R. S., Spira, B. \& Ferreira, R. C. The Pst system of Streptococcus mutans is important for phosphate transport and adhesion to abiotic surfaces. Mol. Oral. Microbiol. 27, 172-181 (2012).

74. Smith, D. J. \& Taubman, M. A. Effect of local deposition of antigen on salivary immune responses and reaccumulation of mutans streptococci. J. Clin. Immunol. 10, 273-281 (1990)

75. Jespersgaard, C. et al. Protective immunity against Streptococcus mutans infection in mice after intranasal immunization with the glucan-binding region of $S$. mutans glucosyltransferase. Infect. Immun. 67, 6543-6549 (1999).

76. Guo, J. H. et al. Construction and immunogenic characterization of a fusion anticaries DNA vaccine against PAC and glucosyltransferase I of Streptococcus mutans. J. Dent. Res. 83, 266-270 (2004).

77. Jiang, H., Hu, Y., Yang, M., Liu, H. \& Jiang, G. Enhanced immune response to a dual-promoter anti-caries DNA vaccine orally delivered by attenuated Salmonella typhimurium. Immunobiology 222, 730-737 (2017).

78. Batista, M. T. et al. LT adjuvant modulates epitope specificity and improves the efficacy of murine antibodies elicited by sublingual vaccination with the N-terminal domain of Streptococcus mutans P1. Vaccine 35, 7273-7282 (2017).

79. Hajishengallis, G., Russell, M. W. \& Michalek, S. M. Comparison of an adherence domain and a structural region of Streptococcus mutans antigen I/II in protective immunity against dental caries in rats after intranasal immunization. Infect. Immun. 66, 1740-1743 (1998).

80. Saito, M. et al. Protective immunity to Streptococcus mutans induced by nasal vaccination with surface protein antigen and mutant cholera toxin adjuvant. $J$. Infect. Dis. 183, 823-826 (2001)

81. Zhao, W., Zhao, Z. \& Russell, M. W. Characterization of antigen-presenting cells induced by intragastric immunization with recombinant chimeric immunogens constructed from Streptococcus mutans Agl/II and type I or type II heat-labile enterotoxins. Mol. Oral. Microbiol. 26, 200-209 (2011).

82. Batista, M. T. et al. Immunogenicity and in vitro and in vivo protective effects of antibodies targeting a recombinant form of the Streptococcus mutans P1 surface protein. Infect. Immun. 82, 4978-4988 (2014).

83. Wunder, D. \& Bowen, W. H. Action of agents on glucosyltransferases from Streptococcus mutans in solution and adsorbed to experimental pellicle. Arch. Oral. Biol. 44, 203-214 (1999).

84. Senpuku, H. et al. Effects of Complex DNA and MVs with GTF extracted from Streptococcus mutans on the oral biofilm. Molecules 24, https://doi.org/10.3390/ molecules24173131 (2019).

85. Morales-Aparicio, J. C. et al. The impacts of sortase A and the 4'-phosphopantetheinyl transferase homolog Sfp on Streptococcus mutans extracellular membrane vesicle biogenesis. Front. Microbiol. 11, 570219 (2020).

86. Liao, S. et al. Streptococcus mutans extracellular DNA is upregulated during growth in biofilms, actively released via membrane vesicles, and influenced by components of the protein secretion machinery. J. Bacteriol. 196, 2355-2366 (2014).

87. Huang, M., Meng, L., Fan, M., Hu, P. \& Bian, Z. Effect of biofilm formation on virulence factor secretion via the general secretory pathway in Streptococcus mutans. Arch. Oral. Biol. 53, 1179-1185 (2008).

88. Harold, F. M. Conservation and transformation of energy by bacterial membranes. Bacteriol. Rev. 36, 172-230 (1972).

89. Konings, W. N., Hellingwerf, K. J. \& Elferink, M. G. The interaction between electron transfer, proton motive force and solute transport in bacteria. Antonie Van Leeuwenhoek 50, 545-555 (1984).

90. Konings, W. N. \& Otto, R. Energy transduction and solute transport in streptococci. Antonie Van Leeuwenhoek 49, 247-257 (1983)

91. Montville, T. J. \& Bruno, M. E. Evidence that dissipation of proton motive force is a common mechanism of action for bacteriocins and other antimicrobial proteins. Int. J. Food Microbiol. 24, 53-74 (1994).
92. Nelson, N. Energizing porters by proton-motive force. J. Exp. Biol. 196, 7-13 (1994).

93. Mitchell, P. Chemiosmotic coupling in oxidative and photosynthetic phosphorylation. 1966. Biochim. Biophys. Acta 1807, 1507-1538 (2011).

94. Nolan, D. P. \& Voorheis, H. P. Hydrogen ion gradients across the mitochondrial, endosomal and plasma membranes in bloodstream forms of trypanosoma brucei solving the three-compartment problem. Eur. J. Biochem. 267, 4601-4614 (2000).

95. Hamilton, I. R. \& St Martin, E. J. Evidence for the involvement of proton motive force in the transport of glucose by a mutant of Streptococcus mutans strain DR0001 defective in glucose-phosphoenolpyruvate phosphotransferase activity. Infect. Immun. 36, 567-575 (1982).

96. Clarkson, J. J. \& McLoughlin, J. Role of fluoride in oral health promotion. Int. Dent. J. 50, 119-128 (2000).

97. Marquis, R. E., Clock, S. A. \& Mota-Meira, M. Fluoride and organic weak acids as modulators of microbial physiology. FEMS Microbiol. Rev. 26, 493-510 (2003).

98. Pandit, S., Kim, H. J., Song, K. Y. \& Jeon, J. G. Relationship between fluoride concentration and activity against virulence factors and viability of a cariogenic biofilm: in vitro study. Caries Res. 47, 539-547 (2013).

99. Sutton, S. V., Bender, G. R. \& Marquis, R. E. Fluoride inhibition of protontranslocating ATPases of oral bacteria. Infect. Immun. 55, 2597-2603 (1987).

100. Bowen, W. H. \& Hewitt, M. J. Effect of fluoride on extracellular polysaccharide production by Streptococcus mutans. J. Dent. Res. 53, 627-629 (1974).

101. Koo, H., Sheng, J., Nguyen, P. T. \& Marquis, R. E. Co-operative inhibition by fluoride and zinc of glucosyl transferase production and polysaccharide synthesis by mutans streptococci in suspension cultures and biofilms. FEMS Microbiol. Lett. 254, 134-140 (2006).

102. Koo, H. et al. Apigenin and tt-farnesol with fluoride effects on $\mathrm{S}$. mutans biofilms and dental caries. J. Dent. Res. 84, 1016-1020 (2005).

103. Koo, H. et al. Effects of apigenin and tt-farnesol on glucosyltransferase activity, biofilm viability and caries development in rats. Oral. Microbiol. Immunol. 17, 337-343 (2002).

104. Gan, Z. et al. Separation and preparation of 6-gingerol from molecular distillation residue of Yunnan ginger rhizomes by high-speed counter-current chromatography and the antioxidant activity of ginger oils in vitro. J. Chromatogr. $B$ Anal. Technol. Biomed. Life Sci. 1011, 99-107 (2016).

105. Hofstetter, R., Fassauer, G. M. \& Link, A. Supercritical fluid extraction (SFE) of ketamine metabolites from dried urine and on-line quantification by supercritical fluid chromatography and single mass detection (on-line SFE-SFC-MS). J. Chromatogr. B Anal. Technol. Biomed. Life Sci. 1076, 77-83 (2018).

106. Morales, D., Piris, A. J., Ruiz-Rodriguez, A., Prodanov, M. \& Soler-Rivas, C. Extraction of bioactive compounds against cardiovascular diseases from Lentinula edodes using a sequential extraction method. Biotechnol. Prog. 34, 746-755 (2018).

107. Najafian, L. \& Babji, A. S. A review of fish-derived antioxidant and antimicrobial peptides: their production, assessment, and applications. Peptides 33, 178-185 (2012).

108. Williams, S., Oatley, D. L., Abdrahman, A., Butt, T. \& Nash, R. Membrane technology for the improved separation of bioactive compounds. Procedia Eng. 44, https://doi.org/10.1016/j.proeng.2012.09.064 (2012).

109. Yoshioka, T., Nagatomi, Y., Harayama, K. \& Bamba, T. Development of an analytical method for polycyclic aromatic hydrocarbons in coffee beverages and dark beer using novel high-sensitivity technique of supercritical fluid chromatography/mass spectrometry. J. Biosci. Bioeng. 126, 126-130 (2018).

110. Zhang, L. et al. [Application of membrane separation technology in extraction process of Chuanxiong Chatiao granules]. Zhongguo Zhong Yao Za Zhi 37, 934-936 (2012).

111. He, G., Yin, Y., Yan, X. \& Wang, Y. Semi-bionic extraction of effective ingredient from fishbone by high intensity pulsed electric fields. J. Food Process Eng. 40, e12392 (2017).

Open Access This article is licensed under a Creative Commons Attribution 4.0 International License, which permits use, sharing, adaptation, distribution and reproduction in any medium or format, as long as you give appropriate credit to the original author(s) and the source, provide a link to the Creative Commons license, and indicate if changes were made. The images or other third party material in this article are included in the article's Creative Commons license, unless indicated otherwise in a credit line to the material. If material is not included in the article's Creative Commons license and your intended use is not permitted by statutory regulation or exceeds the permitted use, you will need to obtain permission directly from the copyright holder. To view a copy of this license, visit http://creativecommons. org/licenses/by/4.0/.

(c) The Author(s) 2021 